\title{
ANÁlISIS DE INDICADORES DE CALIDAD, DESEMPEÑO Y GOBERNANZA DE LOS PEQUEÑOS PRESTADORES A PARTIR DE LA EVALUACIÓN REALIZADA POR LA SSPD DE COLOMBIA
}

\author{
ANALYSIS OF THE QUALITY, PERFORMANCE AND GOVERNANCE \\ INDICATORS FROM THE SMALL PROVIDERS ON THE EVALUATION \\ CONDUCTED BY THE SSPD OF COLOMBIA
}

\author{
Cesar A. Figueredo ${ }^{1}$, Nidia Stella Rincón ${ }^{2}$, Nidia S. Salazar ${ }^{3}$ \\ Escuela de Ciencias Básicas Tecnologías e Ingenierias, Universidad Nacional Abierta y a Distancia, UNAD
}

Recibido: 11/04/2017 • Aprobado: 25/05/2017

\section{RESUMEN}

Los pequeños prestadores de los servicios públicos domiciliarios de acueducto y alcantarillado, surgen a partir de la Ley 142 de 1994, donde se les permite organizarse como: juntas administradoras, juntas de acción comunal, asociaciones de usuarios y entidades cooperativas, regulados y vigilados por entes gubernamentales cuyas funciones son inherentes a la gestión, control y regulación del recurso hídrico, como la Superintendencia de Servicios Públicos Domiciliarios (SSPD), el Ministerio de Vivienda Ciudad y Territorio, Comisión de Regulación de Agua Potable y Saneamiento Básico (CRA). Los pequeños prestadores son organizaciones solidarias y de participación ciudadana, que buscan la autogestión en la prestación del servicio de agua potable, en zonas donde no hay cobertura y que impactan positivamente en el desarrollo socioeconómico de su comunidad; atienden a menos de 2.500 usuarios; por su tamaño generalmente presentan dificultades en su operación, debido a la falta de estructuras tarifarias adecuadas, bajos recaudos que no permiten cubrir los costos de operación y en algunos casos prestar un servicio eficiente y de calidad. Este artículo busca identificar cómo se encuentran los indicadores de calidad del servicio y gobernanza en los acueductos de pequeños prestadores en Colombia de acuerdo a los informes de evaluación de gestión realizados por parte de la SSPD. Los resultados del análisis realizado, a partir de una investigación exploratoria con base en 362 informes que reposan en la SSPD, muestran que los pequeños prestadores encuentran su mayor dificultad en el cumplimiento de los requisitos legales, y la ausencia de los entes gubernamentales frente al apoyo requerido para la atención a estas comunidades.

\footnotetext{
Nomenclatura

SSPD: Superintendencia de Servicios Públicos Domiciliarios

CRA: Comisión de Regulación de Agua Potable

RUPS: Registro Único de Prestadores

IRCA: Índice de Riesgo de Calidad del Agua

${ }^{1}$ cesar.figueredo@unad.edu.co, orcid.org/0000-0002-7381-0042

${ }^{2}$ nidia.rincon@unad.edu.co, orcid.org/0000-0002-5956-6610

${ }^{3}$ nubia.salazar@unad.edu.co, orcid.org/0000-0003-1309-4942
}

PUEAA: Programa de Uso Eficiente de Ahorro de Agua

SUI: Sistema Único de Información. 
Palabras clave: abastecimiento de agua, calidad del agua, gestión, pequeños prestadores, recursos hidricos.

\section{Abstract}

Small public service providers emerged from the Law 142 of 1994, in which they are allowed to organize themselves as: administrative boards, community action boards, user associations and cooperative entities, regulated and monitored by government entities which functions are inherent to the management, control and regulation of the water resource, such as the "Superintendencia de Servicios Públicos Domiciliarios" (SSPD), Ministerio de Vivienda Ciudad y Territorio, Comisión de Regulación de Agua Potable y Saneamiento Básico (CRA). The small public service providers are solidarity organizations and formed by citizen participation, who seeks for self-management in the provision of drinking water service in areas where there is no coverage and that have a positive impact on the socio-economic development of the community; they serve less than 2500 users, because of their size they generally present difficulties in their operation due to the lack of suitable tariff structures, low incomes that cannot afford operating costs and in some cases neither provide an efficient and quality servicehis article seeks to identify how the of service, quality and governance indicators are in aqueducts of small public service providers in Colombia according to the management evaluation reports made by the SSPD, to know the sustainability of these aqueducts. The results of the analysis, made from an exploratory research based on 362 reports that rest in the SSPD; show that small public service providers find their greatest difficulty in the compliance of the legal requirements, and a great absence of government.

Key words: abastecimiento de agua, calidad del agua, gestión, pequeños prestadores, recursos hídricos.

\section{INTRODUCCIÓN}

El agua potable es un recurso vital para la vida humana, tanto en cantidad como en calidad; según Arévalo (2012) citado por Vanegas, Vera \& Torres Ortega (2014), Colombia es uno de los países que posee una gran riqueza en biodiversidad y fuentes hídricas, y uno de los diez con mayor disponibilidad de recurso hídrico en el mundo.

Por otra parte, la calidad del agua en Colombia se ha convertido en una temática con mayor relevancia en la última década, de tal manera, que el acceso a agua potable y el saneamiento han aumentado significativamente en comparación con años anteriores (López, Martínez \& Almario, 2016).

La utilización del recurso hídrico para la satisfacción de las necesidades básicas requiere una calidad ambiental y sanitaria estricta del agua para el consumo humano que permita el mejoramiento de las condiciones de vida y, por ende, la reducción de enfermedades.
(Díaz, Gómez \& Quijano). En ese sentido, es importante resaltar que la calidad del agua para consumo humano es un factor determinante en la salud pública.

En Colombia, las deficientes condiciones sanitariasasociadas a la pobreza y al deterioro ambiental, al igual que las falencias en la prestación del servicio y la falta de cobertura del mismo, ha generado que en zonas urbanas alejadas o en zonas rurales se incremente el porcentaje de enfermedades causadas por el consumo de agua contaminada. (MSPS, 2013 citado por López, Martínez \& Almario, 2016).

A partir de la constitución de 1991 y la expedición de la Ley 142 de 1994 se formaliza a los pequeńos prestadores como organizaciones comunitarias autorizadas para prestar el servicio de agua potable en las zonas alejadas de los centros urbanos; hacen parte de los pequeños prestadores, los acueductos rurales, los cuales funcionan gracias a la gestión y trabajo de sus 
comunidades y se proyectan como una posible solución que garantice el acceso de agua potable.

En América Latina los acueductos rurales surgieron hace más de 40 años, de la necesidad de mejorar en la población rural la calidad de vida y garantizar el acceso a agua potable.

América Latina es una de los territorios que cuenta con mayor recurso hídrico, pero cerca de 34 millones de personas aún no tienen acceso a esta. En franjas distantes donde la situación es más sentida: 21 millones de latinoamericanos que viven en estos territorios no cuen$\tan$ con un servicio adecuado de agua potable y $46 \mathrm{mi}$ llones no disponen de infraestructuras de saneamiento básico. Lo que conduce a situaciones difíciles para la salud, el rendimiento económico y bienestar de las colectividades rurales; generándose alto nivel de exposición a situaciones precarias de esta comunidad, constituida por indígenas y afrodescendientes. (CAF, 2017).

Colombia descentraliza y lleva esta responsabilidad a nivel departamental y municipal, fomentando la creación de empresas que pudieran cumplir con este importante objetivo de cobertura. Este proceso de descentralización se formaliza con la Constitución de 1991 y la expedición de la Ley 142 de 1994, las cuales consolidan el papel de los municipios como responsables de garantizar la prestación de los servicios de agua y saneamiento.

En Colombia el abastecimiento de agua potable, así como la disposición de residuos sólidos y aguas residuales en zonas rurales estuvieron a cargo de la $\mathrm{Na}$ ción hasta los años ochenta, a partir de la descentralización político administrativa, con el Decreto No 077 de 1987 y la expedición de la Constitución Política de 1991 y la Ley 142 de 1994, pasaron a estar bajo la responsabilidad de las administraciones municipales. (DPN, 2014).

Estas organizaciones son responsables de garantizar la sostenibilidad de los servicios de agua potable que brindan en sus zonas de influencia.
En Colombia cerca de doce mil comunidades organizadas prestan los servicios públicos domiciliarios, como agua potable y saneamiento básico a cerca del $40 \%$ de los pobladores rurales en el país. Son estructuras sociales organizadas que han sobrepasado los cambios de carácter político y administrativo, por largo tiempo, en las que se les olvida y reduce. Siendo construidos por decisión de las colectividades para solucionar los problemas que ni el Estado, ni el mercado, logran corregir. (Moncada, 2015)

Las organizaciones comunitarias nacen ante la incapacidad de los entes estatales de suministrar agua potable a su comunidad, y ante la prioridad de cubrir esta necesidad básica.

Los acueductos comunitarios se han afianzado como las tácticas más usadas en zonas más alejadas para que la comunidad pueda suplir la necesidad del recurso hídrico. Son empresas que no buscan generar ganancias, comunes y solidarias que se ha sostenido en el tiempo. Nacen debido a reducción de cantidad y calidad del recurso en sus zonas. (UICN, 2015).

De igual manera, los pequeños prestadores rurales brindan acceso al agua potable a gran parte de la comunidad en su área de influencia, estos acueductos se han venido organizando para estructurar acciones conjuntas en busca de su fortalecimiento y reconocimiento.

Para el año 2015 se conoció que 11 millones 700 mil personas viven en el campo, quienes reciben el servicio de agua potable por parte de 15.000 acueductos rurales, información suministrada en el II Congreso Internacional de Acueductos Rurales y Municipios Prestadores Directos. De los 15.000 acueductos solo 1.465 están registrados ante la SSPD, y están inscritos en el Registro Único de Prestadores, RUPS. (El Colombiano, 2015).

Los pequeños prestadores son comunidades organizadas solidariamente y de participación ciudadana que buscan la autogestión en la prestación del servicio de agua potable en las zonas donde no hay cobertura, la gestión de estas organizaciones impacta positivamente en el desarrollo socioeconómico de su comunidad. 
El valor de la administración, dirección, atención y manejo del recurso hídrico por parte de las colectividades, han evidenciado a través del tiempo una muestra de un conocimiento y conciencia de la atención, conservación y razonamientos de ecuanimidad en su repartición. Es así como el manejo colectivo del recurso hídrico a personalizado unas estructuras de colaboración que implican una reflexión y evaluación sobre las acciones a todo nivel, lo cual ha generado que los acueductos comunitarios trabajen a fin de suplir las insuficiencias y solicitudes de la comunidad, logrando impactar de manera efectiva en el abastecimiento de sus áreas, visionando autorregulación, solidaridad, obligación y un gran sentido de pertenencia por la salvaguardia del agua. (Ojeda, 2015).

En ese orden de ideas, a pesar de los grandes esfuerzos realizados por los pequeños prestadores, estos presentan muchas dificultades como: bajos niveles de inversión, falta de asistencia técnica, falta de apoyo gubernamental, no formalización, falta de abastecimiento al $100 \%$ de los usuarios y falta de continuidad, en esa dirección se hace necesario comprender ¿Cómo se encuentran los indicadores de calidad del servicio, desempeño y gobernanza en los acueductos pequeńos prestadores de acuerdo al informe de la evaluación de gestión por parte de la SSPD de Colombia?

Este artículo se fundamenta en una investigación exploratoria, a partir de los informes de la evaluación a la gestión de los pequeños prestadores en Colombia, es decir aquellos que cuentan con menos de 2.500 suscriptores, realizada por la SSPD quien es la encargada de llevar a cabo las evaluaciones integrales a las empresas prestadoras de dichos servicios públicos; allí reposan 362 informes, organizados por departamentos, siendo estos los estratos para el muestreo; tomándose para este artículo una muestra de 61 acueductos como resultado de utilizar la fórmula de población finita con un nivel de confianza del $95 \%$, valor de $Z=1.96, p=0.95$ y q= 0.05 , un error permitido de e $=0.05$, y la aplicación del muestreo estratificado proporcional.
A la muestra seleccionada se le evaluaron los criterios de calidad del servicio que incluyen los indicadores de cobertura, continuidad, cantidad e Índice de riesgo de calidad del agua (IRCA). El criterio de desempeño y gobernanza en el cual se trabaja los indicadores de gobernanza interna y legalidad, gestión administrativa y gestión técnica operativa. (Smits et al., 2012)

La anterior evaluación se realizó de acuerdo a la valoración de los indicadores propuestos por Smits, Tamayo, Ibarra, Rojas, Benavidez \& Bey en el año 2012, en el estudio titulado: "Gobernanza y sostenibilidad de los sistemas de agua potable y saneamiento rurales en Colombia” del Banco Interamericano de Desarrollo.

El objetivo de este análisis es conocer cómo se encuentran los indicadores de calidad, desempeńo y gobernanza de los pequeños prestadores antes mencionados, donde los resultados obtenidos sirvan de referencia estructural y contextual para posteriores investigaciones.

El artículo está organizado en cuatro partes, la primera parte contiene el marco teórico y normativo de los pequeños prestadores, la segunda presenta el fundamento teórico de los indicadores de sostenibilidad, la tercera el análisis de los resultados y finalmente las conclusiones del estudio.

\section{MARCO TEÓRICO Y NORMATIVO}

El artículo 15 de la Ley 142 de 1994 indica que en Colombia podrán prestar servicios públicos domiciliarios las entidades descentralizadas y las organizaciones autorizadas, las cuales, a su vez, son llamados pequeños o menores prestadores de acuerdo con el Decreto 2590 del 6 de julio de 2007 (DNP, 2007), sí son:

“i) empresas de servicios públicos de acueducto, alcantarillado y aseo que atienden menos de dos mil quinientos (2.500) usuarios;

ii) personas naturales o jurídicas que produzcan para ellas los bienes y servicios propios del objeto de 
las empresas de servicios públicos de acueducto, alcantarillado y aseo;

iii) empresas de servicios públicos de acueducto, alcantarillado y aseo que operen exclusivamente en uno de los municipios clasificados como menores, según la ley, o en zonas rurales;

iv) organizaciones autorizadas de las que trata el numeral 15.4 del artículo 15 de la Ley 142 de 1994 para la prestación de los servicios públicos de acueducto, alcantarillado y aseo;

v) productores marginales de los servicios de acueducto, alcantarillado y aseo;

vi) municipios menores de categorías $5^{\mathrm{a}}$ y $6^{\mathrm{a}} \mathrm{de}$ acuerdo con la Ley 617 de 2000, que sean prestadores directos de los servicios de acueducto, alcantarillado y aseo".

Hacen parte de los pequeños prestadores, los acueductos rurales, los cuales funcionan gracias a la gestión y trabajo de sus comunidades y se proyectan como una posible solución que garantice el acceso de agua potable, si a sus iniciativas y a su conocimiento empírico se le presta la debida atención por partes de los entes gubernamentales, así como se plantea el periódico El Nuevo Diario:

En Latinoamérica, los acueductos rurales pueden llegar a convertirse en la solución al problema de cobertura de agua potable si los Gobiernos los tienen en cuenta a la hora de tomar decisiones sobre las políticas que implican el uso de este importante recurso, igualmente es importante una apropiada capacitación de sus representantes legales. (El Nuevo Diario, 2014).

Es fundamental comprender a su vez que los acueductos rurales no cubren solamente la parte de acueducto y saneamiento básico, sino que también propenden por garantizar agua de calidad en el desarrollo de las actividades agropecuarias. En el mismo sentido Ojeda afirma:
Los acueductos comunitarios juegan un papel preponderante en el contexto de la seguridad y soberanía alimentaria y en la sostenibilidad a nivel local y regional, en primer lugar, por cuanto son fundamentales para fortalecer la cultura campesina y la producción de alimentos para la comunidad, y los mercados regionales y así mismo generar ingresos para el desarrollo de sus comunidades. (Ojeda, 2014).

Con la Constitución Política Colombiana del 91 nace la posibilidad de que los pequeńos prestadores puedan acceder al servicio de agua potable y saneamiento básico a través de la organización de la misma comunidad; conformándose en entes legalmente constituidos de diferente naturaleza jurídica; sin ánimo de lucro, administrados por integrantes de la misma comunidad, regulados y vigilados por entes gubernamentales cuyas funciones son inherentes a la gestión, control y regulación del recurso hídrico, como la SSPD, Ministerio de Vivienda Ciudad y Territorio, y la CRA.

Los pequeños prestadores surgen a partir de la Ley 142 de 1994, donde se les permite organizarse como comunidad para la prestación de servicios públicos y se ratifica en el decreto 421 de 2000; Para ello existen varias figuras organizativas: juntas administradoras, juntas de acción comunal, asociaciones de usuarios y entidades cooperativas. Todas las figuras organizativas permiten conformar un ente administrador, autónomo en sus decisiones y goza de reconocimiento jurídico.

Dentro de las figuras organizativas existen varias formas de constitución contempladas en la normatividad vigente. El decreto 421 de 2000, reglamenta el numeral 4 del art. 15 de la ley 142 de 1994 en relación con las organizaciones autorizadas para prestar los servicios públicos de agua potable y saneamiento básico en municipios menores, zonas rurales y áreas urbanas específicas.

Estos esquemas asociativos corresponden a cualquier tipo de asociación constituida de acuerdo con 
las normas vigentes, en la que participen administradores de punto de suministro o abastos de agua, o prestadores de servicios de acueducto, alcantarillado o aseo, con el objeto de apoyar el acceso al agua potable y al saneamiento básico.

A continuación, se presenta la conceptualización de algunas de estas figuras organizativas (AMAC, s.f.).

Acción comunal: es una expresión social organizada, autónoma y solidaria de la sociedad civil, cuyo propósito es promover un desarrollo integral, sostenible y sustentable construido a partir del ejercicio de la democracia participativa en la gestión del desarrollo de la comunidad.

Junta de Acción Comunal: es una organización cívica, social y comunitaria de gestión social, sin ánimo de lucro, de naturaleza solidaria, con personería jurídica y patrimonio propio, integrada voluntariamente por los residentes de un lugar que aúnan esfuerzos y recursos para procurar un desarrollo integral, sostenible y sustentable con fundamento en el ejercicio de la democracia participativa. Las juntas de acción comunal, son organismos de acción comunal de primer grado.

Asociación de Juntas de Acción Comunal: organismo de acción comunal de segundo grado, tienen la misma naturaleza jurídica de las juntas de acción comunal y se constituye con los organismos de primer grado fundadores y los que posteriormente se afilien.

Federación de Acción Comunal: organismo de acción comunal de tercer grado. Tiene la misma naturaleza jurídica de las juntas de acción comunal y se constituye con los organismos de acción comunal de segundo grado fundadores y los que posteriormente se afilien.

Confederación Nacional de Acción Comunal: organismo de acción comunal de cuarto grado. Tiene la misma naturaleza jurídica de las juntas de acción comunal y se constituye con los organismos de acción comunal de tercer grado fundadores y los que posteriormente se afilien.
Acueductos comunitarios: son construcciones complejas en sentido histórico, social, económico e institucional. Construcciones públicas regionales y locales de gestión del recurso del agua, las cuales se apoyan y al mismo tiempo expresan y recrean los sistemas culturales propios de las comunidades gestoras de las mismas, descendientes o vecinas de los pueblos indígenas actuales o remotos. Hacen parte de los territorios sociales en veredas, resguardos indígenas, territorios de comunidades negras y barrios de las diferentes regiones y ciudades del país. (Superservicios, 2014).

A partir de la necesidad sentida de una población concentrada geográficamente en zonas alejadas de las cabeceras municipales sin acceso al recurso hídrico, la misma comunidad en una dinámica colectiva en búsqueda de solución, se organiza con recursos propios, convirtiéndose en organización estructurada y con reconocimiento jurídico, obteniendo visibilización a nivel nacional, integrando la Red Nacional de Acueductos Comunitarios de Colombia, la cual autodefine los acueductos comunitarios como:

Son organizaciones constituidas por un grupo de vecinos con el objetivo de construir un acueducto con el cual garantizar el acceso y suministro de agua potable, y en algunos casos prestar también el servicio de saneamiento básico. Estos acueductos se constituyen como una organización sin ánimo de lucro, regidas bajo políticas de cooperación y confianza mutua, con el objetivo de beneficiar a sus comunidades y lograr el bienestar para sus territorios. (Minvivienda, s.f.)

Estas organizaciones han adquirido una gran relevancia porque proveen agua potable y saneamiento básico a aquellas comunidades que por su ubicación no había sido posible el cubrimiento del servicio por parte de los entes estatales y, además, porque se están convirtiendo en promotores de mejores condiciones de calidad de vida, en este mismo sentido:

Los acueductos comunitarios son un medio de aumentar el potencial de ajuste de los territorios al cambio climático. En torno a ellos se instaura institucionalidad, 
responsabilidades, colaboración, observación social, control del recurso hídrico y, asimismo, se consolida el conocimiento de las poblaciones. Las acciones en los acueductos comunitarios originan colectividades autogestionarias, competentes de establecer y de planear sus áreas en función del agua y del avance regional. (CRA, s.f.).

Las organizaciones anteriormente mencionadas, y en concordancia con la importante participación e impacto que están teniendo en la solución del cubrimiento de una de sus necesidades básicas y dada la evolución que vienen desarrollando, se hizo imperioso tanto para el gobierno como para las mismas organizaciones, el acompañamiento técnico, administrativo y financiero, así como el seguimiento, control, y coordinación de las actividades propias de los acueductos veredales.

El Estado cuenta con entidades cuya función están a la luz del marco normativo colombiano. En la Tabla 1 , se presentan algunas de estas entidades y sus respectivas funciones.

\section{TABla 1}

Entidades gubernamentales

\begin{tabular}{|l|l|}
\hline \multicolumn{1}{|c|}{ Entidad } & \multicolumn{1}{c|}{ Función } \\
\hline $\begin{array}{l}\text { Superintendencia de } \\
\text { Servicios Públicos } \\
\text { Domiciliarios SSPD }\end{array}$ & $\begin{array}{l}\text { Genera los insumos en el análisis y formulación de políticas sectoriales, obtenidos a partir de la in- } \\
\text { formación reportada al Sistema Unico de Información (SUI), son análisis en los aspectos financieros, } \\
\text { técnicos, comerciales y administrativos, que forman parte de las evaluaciones integrales a las empresas } \\
\text { prestadoras de servicios públicos, realizadas por parte de la Superintendencia Delegada para Acueduc- } \\
\text { to, Alcantarillado y Aseo de la Superintendencia de Servicios Públicos Domiciliarios (Presidencia de la } \\
\text { República, 2016). }\end{array}$ \\
\hline $\begin{array}{l}\text { Ministerio de Vivienda } \\
\text { Ciudad y Territorio }\end{array}$ & $\begin{array}{l}\text { Dentro de sus funciones está coordinar y articular con el Ministerio de Agricultura y Desarrollo } \\
\text { Rural; la formulación de proyectos, programas, adopción de políticas que permitan el acceso de agua } \\
\text { potable y saneamiento básico a la población de las zonas rurales (CRA, 2015). }\end{array}$ \\
\hline $\begin{array}{l}\text { Comisión de Regulación } \\
\text { de Agua Potable y } \\
\text { Saneamiento Básico - } \\
\text { CRA }\end{array}$ & $\begin{array}{l}\text { Como entidad de orden nacional, su propósito fundamental es regular los monopolios, promover } \\
\text { la competencia, impulsar la sostenibilidad del sector Agua Potable y Saneamiento Básico, evitando } \\
\text { arbitrariedades de posición dominante, garantizando la prestación de servicios de calidad, con tarifas } \\
\text { razonables y vasta cobertura; contribuyendo al bienestar de la población y a la mejora de las condicio- } \\
\text { nes del mercado (Congreso de Colombia 2002). }\end{array}$ \\
\hline
\end{tabular}

Fuente: Smits et al., 2012.

\section{INDICADORES}

Una forma de analizar el bien quehacer de los acueductos comunitarios es a través de los indicadores de calidad de agua, desempeño y gobernanza, que para el presente estudio se entiende como el contar con la infraestructura necesaria, los recursos humanos, financieros, hídricos y físicos que garanticen el funcionamiento del sistema a lo largo del tiempo, logrando ofrecer un servicio de agua potable de calidad.

Teniendo en cuenta el anterior fundamento y basados en el estudio de gobernanza y sostenibilidad de los sistemas de agua potable y saneamiento rurales en Colombia del BID, se realiza el análisis de los indicadores en dos grandes grupos:

El primero en Calidad del agua que incluye la cobertura, continuidad, calidad y cantidad. El segundo, Gobernanza que incluye gobernanza interna y legalidad, gestión administrativa, y gestión técnica operativa, los cuales están agrupados y conceptuados en la Tabla II. 


\section{Análisis de resultados}

\subsection{Calidad del servicio}

Los resultados obtenidos se evalúan a partir de los dos grandes grupos establecidos en la Tabla 2, calidad de servicio y gobernanza con sus respectivas divisiones. La calificación que se le dio a cada ítem, en el grupo calidad del servicio, se valoró a partir de los puntajes contemplados en la Escalera del agua (DNP, 2007, 27).

A su vez en el grupo de gobernanza se hizo la evaluación a partir de la calificación para indicadores gobernanza y desempeño del prestador (DNP, 2007, 39).

La calidad del servicio representa las características con las cuales los usuarios o suscriptores reciben el mismo, y, la percepción del usuario sobre estas variables en forma de su satisfacción con el servicio (DNP, 2007, 26).
En el criterio de cobertura se tiene que el $59 \%$ de los acueductos están atendiendo a más del $90 \%$ de los suscriptores frente a los potenciales que deben atenderse, obteniendo una calificación alta, por el contrario, el $41 \%$ de los acueductos no están logrando llegar a más del $90 \%$ de los suscriptores, lo que implica que todavía un alto porcentaje de pequeńos prestadores requieren formular estrategias que les garantice poder llegar al $100 \%$ de sus usuarios.

En el indicador de continuidad, que mide el número de horas de prestación del servicio solamente el $68 \%$ de los acueductos analizados presta el servicio en un rango mayor a las 23 horas por día, el $18 \%$ de los pequeños prestadores presta el servicio en un rango inferior entre 12 y 19 horas por día, mientras que el $15 \%$ presta el servicio por menos de 12 horas por día, para los casos contemplados en este último porcentaje los informes generados por la superintendencia de servicios públicos no permiten evidenciar las causas de estos bajos niveles de continuidad.

\section{TABLA 2}

Indicadores

\begin{tabular}{|c|c|c|}
\hline \multicolumn{2}{|c|}{ Indicador } & Concepto \\
\hline \multirow{6}{*}{$\begin{array}{l}\text { Calidad } \\
\text { del servicio }\end{array}$} & Cobertura & $\begin{array}{l}\text { Se precisa como la representación a los beneficiarios servidos (residencias inscritas en la pres- } \\
\text { tación del servicio), de cara a la capacidad del servicio del procedimiento, la cual está explícita } \\
\text { por el número de residencias en la colectividad y por el compromiso definido por términos } \\
\text { geográficos. }\end{array}$ \\
\hline & Continuidad & Se especifica como el número de horas al día que se proporciona la prestación \\
\hline & Cantidad & $\begin{array}{l}\text { Es la suma total del recurso hídrico que alberga cada consumidor en la residencia, } \\
\text { mencionado en litros por persona por ciclo diario. }\end{array}$ \\
\hline & Índice de Riesgo & $\begin{array}{l}\text { IRCA calcula el nivel de riesgo de adquirir padecimientos concernientes con el gasto } \\
\text { de recurso hídrico potable. }\end{array}$ \\
\hline & $\begin{array}{l}\text { Agua para consu- } \\
\text { mo Humano }\end{array}$ & $\begin{array}{l}\text { Para hallar el IRCA se fija un puntaje de peligro a un conjunto de peculiaridades o } \\
\text { cuantificaciones fundamentales del agua (físicos, químicos y microbiológicos que se examinan } \\
\text { en el laboratorio) de una pauta que se hallen por fuera de lugar de las categorías de aprobación }\end{array}$ \\
\hline & $\begin{array}{l}\text { Satisfacción } \\
\text { del usuario }\end{array}$ & $\begin{array}{l}\text { Determinado como la proporción de beneficiarios que están de acuerdo con uno o más uni- } \\
\text { dades de la prestación. Para ello, se realiza una indagación a través de un laboratorio con un } \\
\text { conjunto de la colectividad, donde se evalúa el estado del de agrado en referencia a la calidad, } \\
\text { cantidad, continuidad y la gestión del servicio de abastecimiento de agua. }\end{array}$ \\
\hline $\begin{array}{l}\text { Desempeño } \\
\text { y gobernanza }\end{array}$ & $\begin{array}{l}\text { Gobernanza } \\
\text { interna y legalidad }\end{array}$ & $\begin{array}{l}\text { Característica especifica observable: que muestran el acatamiento de los primeros talantes } \\
\text { normativos, en concordancia con el cerco de exigencias de la Ley } 142 \text { de } 1994 \\
\text { (Ley general de servicios públicos domiciliarios). }\end{array}$ \\
\hline
\end{tabular}




\begin{tabular}{|l|l|l|}
\hline \multicolumn{2}{|c|}{ Indicador } & \multicolumn{1}{c|}{ Concepto } \\
\hline \multirow{2}{*}{$\begin{array}{l}\text { Indicadores } \\
\text { de gestión }\end{array}$} & $\begin{array}{l}\text { Gestión } \\
\text { administrativa }\end{array}$ & $\begin{array}{l}\text { Esta clase contiene variables específicas que evalúan la labor del generador del servicio en par- } \\
\text { tes de administración, circunscribiendo la administración contable-financiero, el potencial del } \\
\text { prestador para equilibrar los costos de la generación de la actividad comercial, y la formación } \\
\text { del talento humano del prestador. }\end{array}$ \\
\cline { 2 - 3 } & $\begin{array}{l}\text { Gestión } \\
\text { técnica operativa }\end{array}$ & $\begin{array}{l}\text { Gestión técnico operativa: esta clase de características cuantificables acceden a prestar atención } \\
\text { sobre el potencial del generador del servicio con el fin de realizar procesos de mantenibilidad. } \\
\text { De igual manera, este evaluador evidencia una característica relacionada con el contenido del } \\
\text { medio y la naturaleza, primordial para responder a la demanda del agua de la región. }\end{array}$ \\
\hline
\end{tabular}

Fuente: Smits et al., 2012.

\section{TABla 3}

Calidad del Servicio

\begin{tabular}{|c|c|c|c|c|c|c|c|c|}
\hline \multirow{2}{*}{ Calificacion } & \multicolumn{2}{|c|}{ Cobertura } & \multicolumn{2}{|c|}{ Continuidad } & \multicolumn{2}{|c|}{ Calidad } & \multicolumn{2}{|c|}{ Cantidad } \\
\hline & Cant. & $\%$ & Cant. & $\%$ & Cant. & $\%$ & Cant. & $\%$ \\
\hline Alto & 36 & $59 \%$ & 41 & $68 \%$ & 45 & $74 \%$ & 29 & $47 \%$ \\
\hline Aceptable & 7 & $12 \%$ & 0 & $0 \%$ & 9 & $15 \%$ & 5 & $9 \%$ \\
\hline Deficiente & 13 & $21 \%$ & 11 & $18 \%$ & 4 & $6 \%$ & 4 & $6 \%$ \\
\hline Muy deficiente & 5 & $9 \%$ & 9 & $15 \%$ & 4 & $6 \%$ & 23 & $38 \%$ \\
\hline Total & 61 & $100 \%$ & 61 & $100 \%$ & 61 & $100 \%$ & 61 & $100 \%$ \\
\hline
\end{tabular}

Fuente: elaboración propia

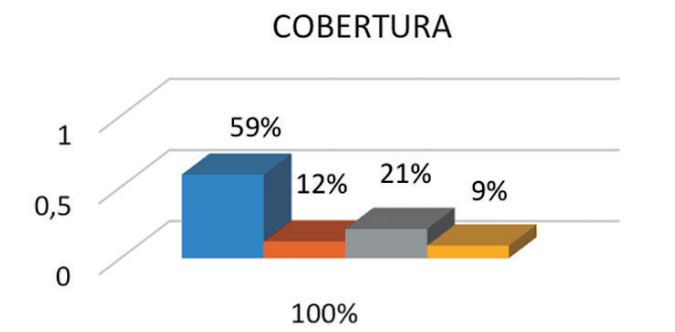

$\square$ ALTO $\square$ ACEPTABLE $\square$ DEFICIENTE $\square$ MUY DEFICIENTE

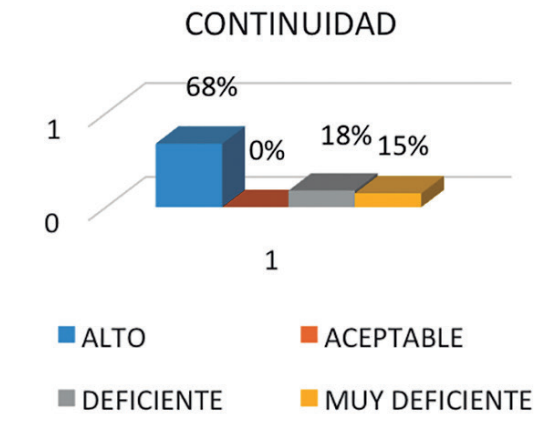

CALIDAD

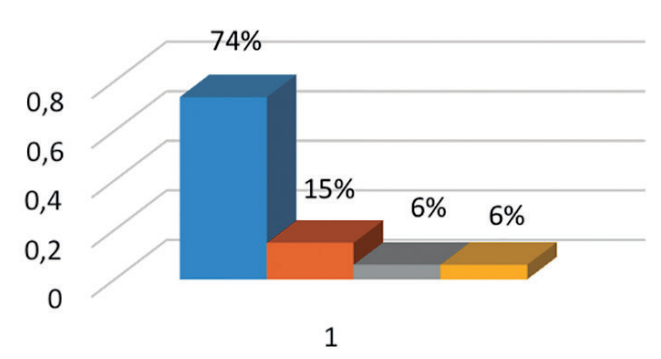

$\square$ ALTO $\square$ ACEPTABLE $\square$ DEFICIENTE $\square$ MUY DEFICIENTE
CANTIDAD

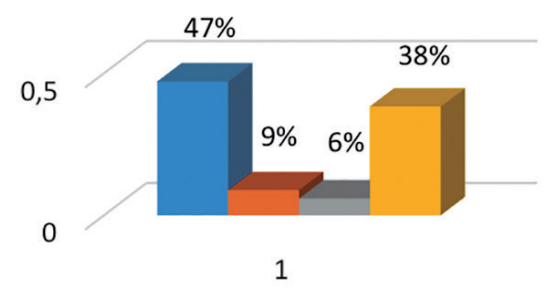

ALTO

- ACEPTABLE

DEFICIENTE

Fig. 1. Indicadores de Calidad del Servicio.

Fuente: elaboración propia 
En el indicador de calidad que representa el nivel de riesgo de contraer enfermedades relacionadas con el consumo de agua potable, se obtuvo que el $74 \%$ de los pequeños prestadores tienen una cualificación alta es decir que su nivel de riesgo se encuentra entre un intervalo de 0 a $5 \%$ (lo que significa: sin riesgo - agua apta para el consumo humano). Sin embargo, el $6 \%$ de los acueductos poseen cualificación muy baja es decir nivel de riesgo muy alto, se encuentran en un intervalo mayor al $80 \%$ de riesgo (lo que significa que se está brindando a los usuarios agua no apta para el consumo humano). Una de las causas evidenciadas en los informes analizados por la SSPD es lavado y desinfección deficiente de la red de distribución.

La importancia de la calidad del agua en Colombia se ha convertido en una temática con mayor relevancia en la última década, de tal manera, el acceso a agua potable y saneamiento han aumentado significativamente en comparación con años anteriores. (López, 2014).

A su vez en el indicador de cantidad, que es la cantidad neta de agua que recibe cada usuario en la vivienda, expresado en litros por habitante por día, se encontró que el $38 \%$ obtuvo una calificación de muy deficiente, lo anterior indica que en estos acueductos no existen datos de macro medición, en ese caso para determinar la información se tomó el caudal promedio de entrada al cual se le resto el $50 \%$ como porcentaje de pérdidas y se dividió por el total de usuarios del sistema. A su vez el $47 \%$ de los acueductos sí cuenta con un sistema de micro medición donde se puede identificar el consumo por usuario, el cual se encuentra en promedio entre 130 y 170 litros/día.

\subsection{Gobernanza interna y legalidad}

Los indicadores de gobernanza interna y legalidad reflejan el cumplimiento de los principales aspectos legales, de acuerdo con el marco de exigencias de la Ley
142 de 1994 (Ley general de servicios públicos domiciliarios). Igualmente se recupera información sobre la estructura organizativa y su operatividad, aspectos de gestión, toma de decisiones y gestión participativa del servicio (DNP, 2007, 30).

En la calificación de aceptable se encuentra el 71 $\%$, lo que significa que este porcentaje de acueductos cumple entre tres y cuatro requisitos (inscripción a la cámara de comercio, inscripción SSPD y reporte al SUI, inscripción y reporte de tarifas a la CRA, tener el permiso o concesión de aguas y tener contrato de condiciones uniformes), exigencias de la Ley 142 de 1994; pero en ninguno de los casos analizados se encontró que alguno esté cumpliendo con el total de los requisitos.

En cuanto a las PQR se tiene que el $47 \%$ se encuentra clasificado como deficiente, lo que significa que este porcentaje de acueductos no cuenta con una oficina y/o proceso para la atención de las PQR de sus usuarios.

En la parte de la estructura organizativa se tiene un $79 \%$ en aceptable, indicando que no existe un organigrama formalmente definido, pero que si se tiene claro cuáles son las funciones y responsabilidades de cada cargo que integra el acueducto.

Para la gestión de la reposición de activos el $85 \%$ de los acueductos están clasificados como deficientes, porque a pesar de tener necesidades en cuanto a esta reposición, no tienen definido la planeación ni los recursos para poder realizarlas.

En las relaciones interinstitucionales la calificación es deficiente con un $94 \%$, es decir que el número de entidades estatales no hacen presencia institucional en cuanto al apoyo requerido por acueductos; se evidenció que cada acueducto recibe menos de tres visitas de las mencionadas por año. 
TABLA 4

Gobernanza interna y legalidad

\begin{tabular}{|c|c|c|c|c|c|c|c|c|c|c|}
\hline \multirow[t]{2}{*}{ CALIFICACIÓN } & \multicolumn{2}{|c|}{$\begin{array}{l}\text { Cumplimiento } \\
\text { de requisitos legales } \\
\text { de los prestadores }\end{array}$} & \multicolumn{2}{|c|}{$\begin{array}{c}\text { Proceso de } \\
\text { Peticiones, Quejas } \\
\text { y Reclamos (PQR) }\end{array}$} & \multicolumn{2}{|c|}{$\begin{array}{c}\text { Estructura } \\
\text { organizativa }\end{array}$} & \multicolumn{2}{|c|}{$\begin{array}{l}\text { Gestión para } \\
\text { reposición de } \\
\text { activos }\end{array}$} & \multicolumn{2}{|c|}{$\begin{array}{c}\text { Relaciones } \\
\text { interinstitucionales }\end{array}$} \\
\hline & CANT. & $\%$ & CANT. & $\%$ & CANT. & $\%$ & CANT. & $\%$ & CANT. & $\%$ \\
\hline Alto & 16 & $26 \%$ & 2 & $3 \%$ & 4 & $6 \%$ & 0 & $0 \%$ & 0 & $0 \%$ \\
\hline Aceptable & 43 & $71 \%$ & 14 & $24 \%$ & 48 & $79 \%$ & 0 & $0 \%$ & 2 & $3 \%$ \\
\hline Deficiente & 2 & $3 \%$ & 16 & $26 \%$ & 9 & $15 \%$ & 9 & $15 \%$ & 57 & $94 \%$ \\
\hline $\begin{array}{l}\text { Muy } \\
\text { deficiente }\end{array}$ & 0 & $0 \%$ & 29 & $47 \%$ & 0 & $0 \%$ & 52 & $85 \%$ & 2 & $3 \%$ \\
\hline Total & 61 & $100 \%$ & 61 & $100 \%$ & 61 & $100 \%$ & 61 & $100 \%$ & 61 & $100 \%$ \\
\hline
\end{tabular}

Fuente: elaboración propia

\subsection{Gestión administrativa}

Esta categoría incluye indicadores que miden el desempeńo del prestador en aspectos de administración, incluyendo el manejo contable-financiero, la capacidad del prestador para recuperar los costos de la prestación de servicios, aspectos comerciales y la capacitación del personal del prestador (DNP, 2007, 30).

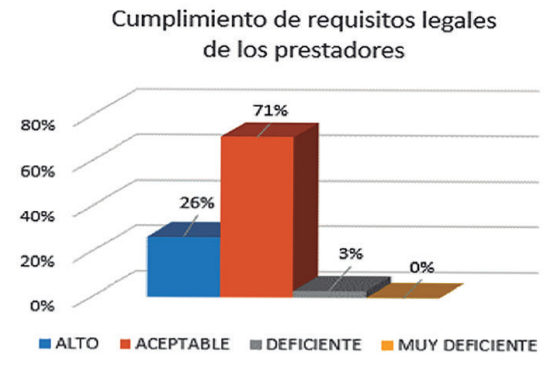

Estructura organizativa

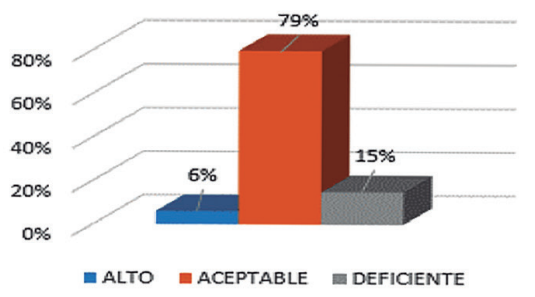

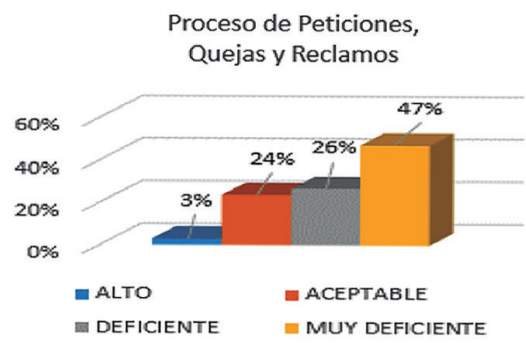

Gestión para reposición de activos

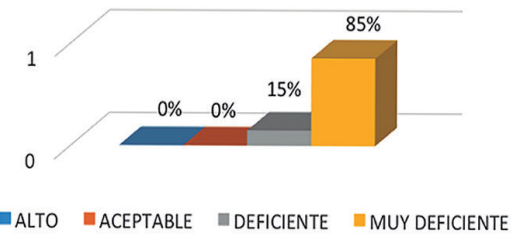

Relaciones interinstitucionales

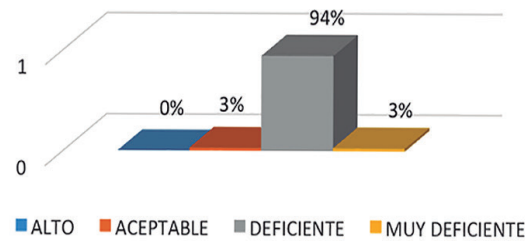

Fig. 2. Indicadores de Gobernanza Interna y legalidad.

Fuente: elaboración propia 
En cuanto a registro de suscriptores se tiene un $53 \%$ como deficiente, indicando que se cuenta con un registro manual de suscriptores y que se actualiza ocasionalmente.

Para la recuperación de cartera se encuentra el $68 \%$ de los acueductos cualificados como aceptable, significando que este porcentaje de acueductos cuenta con al menos un mecanismo o una estrategia para la recuperación del dinero que les adeudan y se está implementando.

\section{Registro de suscriptores}

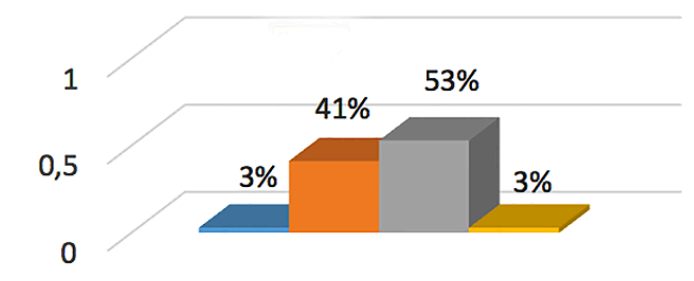

$\square$ ALTO $\square$ ACEPTABLE $\square$ DEFICIENTE $\square$ MUY DEFICIENTE

\section{Metodología tarifaria}

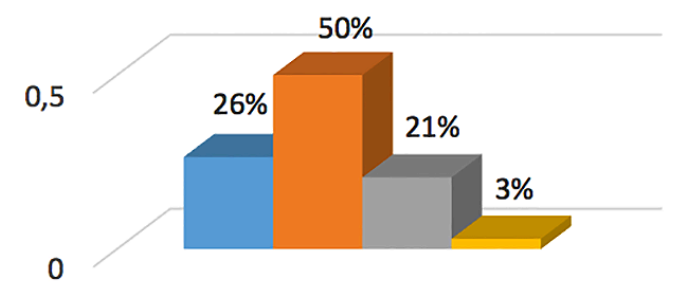

$\square$ ALTO $\square$ ACEPTABLE $\square$ DEFICIENTE $\square$ MUY DEFICIENTE
En el estado de la infraestructura, la calificación es aceptable, es decir el $50 \%$ de los acueductos cuentan con todos los componentes para prestar el servicio.

El $82 \%$ de los acueductos no cuentan con programas formales para protección de la cuenca o la fuente de abastecimiento.

En cuanto al mejoramiento de la calidad del agua el $88 \%$ de los acueductos cuenta con planta de tratamiento de agua potable, la cual está en buenas condiciones y funcionando.

\section{Recuperación de cartera}

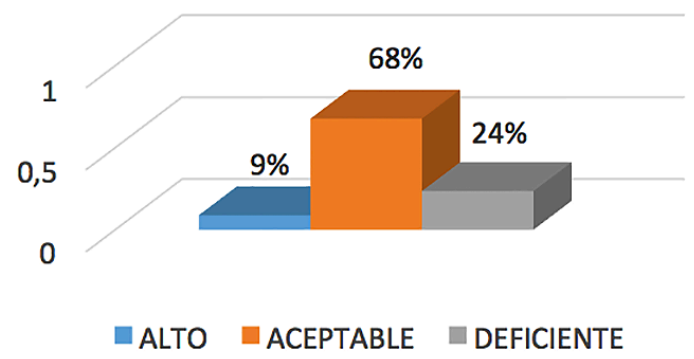

Registros contables básicos

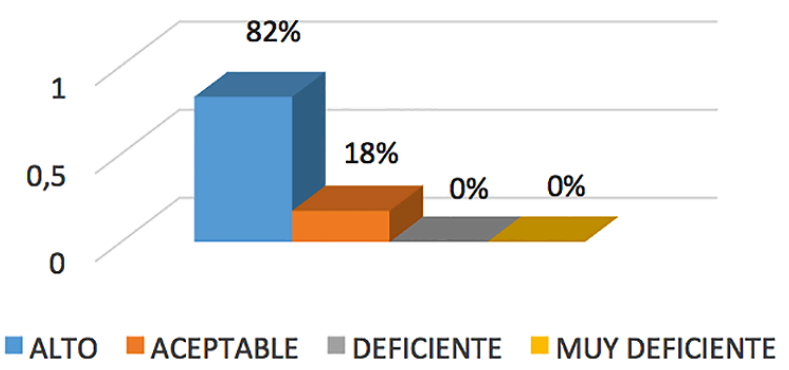

Fig. 3. Indicadores Gestión Administrativa.

Fuente: elaboración propia 
TABLa 5

Gestión administrativa

\begin{tabular}{|l|c|c|c|c|c|c|c|c|}
\hline \multirow{2}{*}{ CALIFICACIÓN } & \multicolumn{2}{|c|}{$\begin{array}{c}\text { Registro } \\
\text { de Suscriptores }\end{array}$} & \multicolumn{2}{c|}{$\begin{array}{c}\text { Recuperación } \\
\text { de Cartera }\end{array}$} & \multicolumn{2}{c|}{$\begin{array}{c}\text { Matolología } \\
\text { Tarifaria }\end{array}$} & \multicolumn{2}{c|}{$\begin{array}{c}\text { Registros Contables } \\
\text { Básicos }\end{array}$} \\
\cline { 2 - 10 } & CANT. & $\%$ & CANT. & $\%$ & CANT. & $\%$ & CANT. \\
\hline Alto & 2 & $3 \%$ & 5 & $9 \%$ & 16 & $26 \%$ & 50 & $82 \%$ \\
\hline Aceptable & 25 & $41 \%$ & 41 & $68 \%$ & 31 & $50 \%$ & 11 & 0 \\
\hline Deficiente & 32 & $53 \%$ & 14 & $24 \%$ & 13 & $21 \%$ & 0 & $0 \%$ \\
\hline Muy deficiente & 2 & $3 \%$ & 0 & $0 \%$ & 2 & $3 \%$ & 0 & 0 \\
\hline Total & 61 & $100 \%$ & 61 & $100 \%$ & 61 & $100 \%$ & 61 & $100 \%$ \\
\hline
\end{tabular}

Fuente: elaboración propia

En la metodología tarifaria está en un $50 \%$ de los acueductos clasificados entonces como aceptables, es decir estos están aplicando la metodología tarifaria de la CRA; es de aclarar que solo se recupera los costos administrativos y operativos, sin lograr un lucro adicional dada la naturaleza de su función.

El $82 \%$ de los acueductos analizados se encuentra en una calificación alta, señalando que los acueductos llevan los registros contables básicos, como libro diario, balance general y estado de resultados.

\subsection{Gestión técnica operativa}

Este grupo de indicadores permite observar la capacidad del prestador para llevar a cabo la operación y mantenimiento del sistema. Así mismo, el indicador captura

una variable relacionada con el tema ambiental, fundamental para garantizar la disponibilidad del recurso hídrico local (DNP, 2007, 30).

TABLA 6

Gestión Técnico Operativa

\begin{tabular}{|l|c|c|c|c|c|c|c|c|c|c|}
\hline \multirow{2}{*}{ Calificación } & \multicolumn{2}{|c|}{$\begin{array}{c}\text { Micromedición } \\
\text { y so eficiente } \\
\text { del agua }\end{array}$} & \multicolumn{2}{|c|}{$\begin{array}{c}\text { Autonomía } \\
\text { operativa }\end{array}$} & \multicolumn{2}{c|}{$\begin{array}{c}\text { Estado de la } \\
\text { infraestructura }\end{array}$} & $\begin{array}{c}\text { Manejo del recurso } \\
\text { hídrico }\end{array}$ & $\begin{array}{c}\text { Mejoramiento de } \\
\text { calidad de agua }\end{array}$ \\
\cline { 2 - 12 } & CANT. & $\%$ & CANT. & $\%$ & CANT. & $\%$ & CANT. & $\%$ & CANT. \\
\hline Alto & 23 & $38 \%$ & 5 & $9 \%$ & 16 & $26 \%$ & 4 & $6 \%$ & 54 & $88 \%$ \\
\hline Aceptable & 18 & $29 \%$ & 41 & $68 \%$ & 31 & $50 \%$ & 7 & $12 \%$ & 5 & $9 \%$ \\
\hline Deficiente & 11 & $18 \%$ & 14 & $24 \%$ & 13 & $21 \%$ & 50 & $82 \%$ & 2 & $3 \%$ \\
\hline Muy deficiente & 9 & $15 \%$ & 0 & $0 \%$ & 2 & $3 \%$ & 0 & $0 \%$ & 0 & $0 \%$ \\
\hline Total & 61 & $100 \%$ & 61 & $100 \%$ & 61 & $100 \%$ & 61 & $100 \%$ & 61 & $100 \%$ \\
\hline
\end{tabular}

Fuente: elaboración propia 


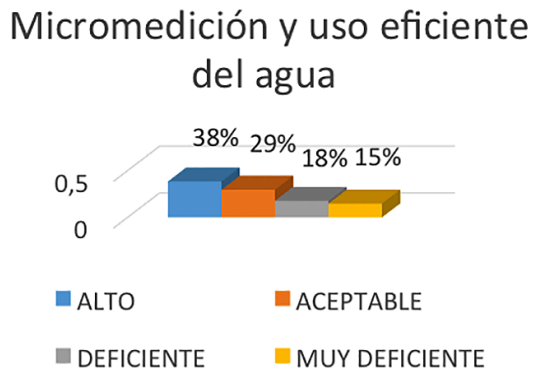

Estado de la infraestructura

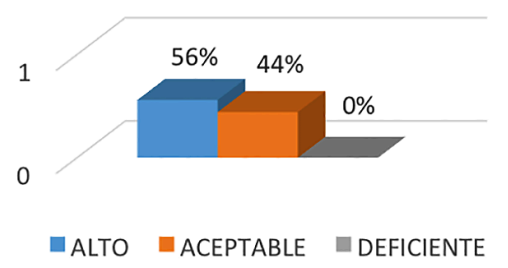

Autonomía operativa

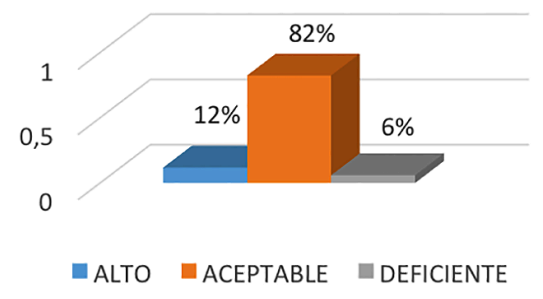

Manejo del recurso hídrico

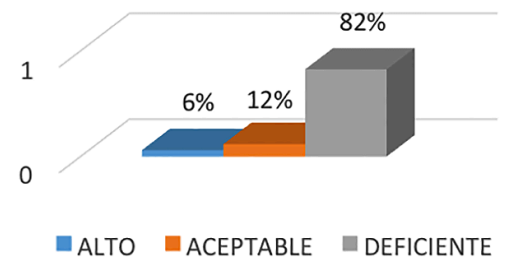

Mejoramiento de calidad de agua

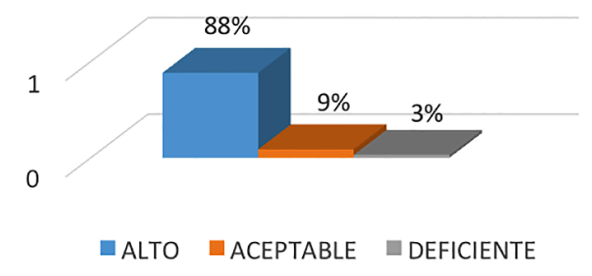

Fig. 4. Indicadores Gestión Técnico Operativa.

Fuente: elaboración propia

El $38 \%$ de los acueductos veredales cuentan con un sistema de micro medición, el cual funciona y se utiliza para el cobro periódico del servicio, fortaleciendo el uso eficiente del recurso.

En la autonomía operativa, el $68 \%$ de los acueductos están cualificados de aceptable, es decir que cuentan con los insumos y recursos para operar de manera autosuficiente.

\section{Conclusiones}

El análisis se realizó en concordancia con la información de la SSPD, quien genera los informes de la evaluación de la gestión de los pequeños prestadores en
Colombia, no obstante, es importante hacer claridad que dentro de los documentos estudiados se puntualiza que no existen registros completos en referencia al IRCA, por tanto, no se puede realizar un análisis fiel a la situación real de los acueductos trabajados. Sin embargo, según los registros obtenidos en este estudio, se estableció que el nivel de riesgo de calidad de agua es bajo.

En relación a la continuidad, los indicadores arrojaron valores que muestran a los pequeños prestadores brindando este servicio por encima de lo mínimo establecido de la norma vigente, es así como el usuario tiene acceso al servicio de agua con un número de horas de prestación mayor a 23 horas por día. 
En la gobernanza de los pequeños prestadores de acueductos, en la gran mayoría de aspectos evaluados se encuentran con una calificación aceptable, es decir no están cumpliendo con todo lo requerido por falta de conocimiento en estos aspectos; se sugiere que el apoyo gubernamental también se enfoque en programas institucionales en las áreas financieras y técnicas, que atienda a los diferentes temas específicos requeridos por cada prestador.

Finalmente se puede concluir que los pequeños prestadores están generando el servicio básico de acueducto, cumpliendo con su quehacer natural y por el cual se constituyeron; pero para ellos no es fácil dar cumplimiento completo de los requisitos emitidos por las entidades gubernamentales por falta de presencia de las mismas, y por falta de unos canales de comunicación efectiva que garantice la doble vía de la información y la retroalimentación sistemática y permanente de los procesos administrativos; a su vez, estos pequeños prestadores tienen dificultades de tipo económico porque su estructura financiera no da alcance para proyectos a largo plazo, sino solo cubre los costos básicos de operación.

\section{REFERENCIAS}

Asociación Municipal de Acueductos Comunitarios de Dosquebradas Risaralda, AMAC. (s.f.). Qué son los acueductos comunitarios. Recuperado en: http://www.amacporelagua.com/ QUESON.html

Arevalo, D. (2012). A look at colombian agriculture from its water footprint. World Wildlife Fundation, WWF. Cali, Colombia, 2012 .

Banco de Desarrollo de América Latina, CAF. (2017). Agua y saneamiento en la nueva ruralidad de América Latina. Recuperado en: https://www.caf.com/es/actualidad/noticias/2017/02/ agua-y-saneamiento-en-la-nueva-ruralidad-de-america-latina/

Comisión de Regulación de Agua Potable y Saneamiento Básico. CRA. (s.f.). Acerca de la entidad: estructura organizacional. Recuperado en: http://www.cra.gov.co/es/acerca-de-la-entidad/ estructura-organizacional

Comisión de Regulación de Agua Potable y Saneamiento Básico (CRA). (2001). Resolución CRA 150 de 2001. Recuperado en: http://www.alcaldiabogota.gov.co/sisjur/normas/Norma1. jsp? $\mathrm{i}=19990$
Comisión de Regulación de Agua Potable y Saneamiento Básico (CRA). (2015). Proyecto de resolución de la CRA 717 de 2015. Recuperado en: http://tramitesccu.cra.gov.co/normatividad/ fichaArchivo. $\operatorname{aspx}$ ?id=2327

Congreso de Colombia. (1994). Ley 142 de 1994. Por la cual se establece el régimen de los servicios públicos domiciliarios y se dictan otras disposiciones. Recuperado en: http://www.alcaldiabogota.gov.co/sisjur/normas/Norma1.jsp?i=2752

Congreso de Colombia. (2002). Ley 743 de 2002. Por la cual se desarrolla el artículo 38 de la Constitución Política de Colombia en lo referente a los organismos de acción comunal. Artículo 6. Recuperado en: http://www.alcaldiabogota.gov.co/sisjur/normas/Norma1.jsp?i=5301

Corte Constitucional Constitución Política de Colombia 1991 (2015). Artículo 365. Recuperado en: http://www.corteconstitucional.gov.co/inicio/Constitucion $\% 20$ politica $\% 20 \mathrm{de} \% 20$ Colombia\%20-\%202015.pdf

Departamento Nacional de Planeación. (2007). Decreto 2590 del 6 de julio de 2007. Por el cual se modifica la estructura de la Superintendencia de Servicios Públicos Domiciliarios. Recuperado en: http://www.creg.gov.co/html/Ncompila/htdocs/Documentos/Energia/docs/decreto_2590_2007.htm

Departamento Nacional de Planeación (2014). Política para el suministro de agua potable y saneamiento básico en la zona rural. Documento Conpes 3810. Recuperado en: http://www.minvivienda.gov.co/conpesagua/3810\%20-\%202014.pdf

Díaz, J., Gómez, N. \& Quijano, A. (2016). Comparación entre los índices de agua potable IAP y los índices de riesgo de la calidad de agua para consumo humano IRCA utilizados para la determinación de la calidad del agua para consumo humano. Revista Especializada en Ingeniería, 4, Recuperado en: http://hemeroteca.unad.edu.co/index.php/publicaciones-e-investigacion/issue/ view/54

El Colombiano. (2015). Los acueductos rurales tendrán vigilancia especial. Recuperado en: http://www.elcolombiano.com/ antioquia/los-acueductos-rurales-tendran-vigilancia-especialBL1951131

El nuevo Diario. (2014). Acueductos rurales, vitales para saciar la sed. Recuperado en: http://www.elnuevodiario.com.ni/ internacionales/326989-acueductos-rurales-vitales-saciar-sed/

López, V. (2014). Propuesta metodológica para el rediseño de una red meteorológica en un sector de la región andina colombiana. Publicaciones e Investigación, 8, 55-76. Recuperado en: http:// hemeroteca.unad.edu.co/index.php/publicaciones-e-investigacion/article/view/1281/1617

López; V., Martínez, J. \& Almario, D. (2016). Análisis del índice de riesgo de la calidad del agua para consumo humano -IRCAy su relación con variables meteorológicas y ubicación geográfica para el departamento del Tolima en los ańos 2012-2013. Revista Especializada en Ingeniería, 10, Recuperado en: http:// hemeroteca.unad.edu.co/index.php/publicaciones-e-investigacion/article/view/161 
Ministerio de Desarrollo Económico. (2000). Resolución 1096 de 2000. Por la cual se adopta el Reglamento Técnico para el sector de Agua Potable y Saneamiento Básico - RAS. Recuperado en: http://www.minvivienda.gov.co/ResolucionesAgua/1096\%20 $-\% 202000 . p d f$

Ministerio de Salud y Protección Social (MSPS). (2013). Informe Nacional Calidad de Agua para Consumo Humano, julio de 2007 - diciembre de 2011. Bogotá: Publicaciones Instituto Nacional de Salud.

Ministerio de Vivienda Ciudad y Territorio de Colombia. (s.f.) Programa Cultura del Agua. Recuperado en: http://www.minvivienda.gov.co/viceministerios/viceministerio-de-agua/programas/cultura-del-agua

Moncada, J. P. (2015). Comunidades organizadas y el servicio público de agua potable en Colombia: una defensa de la tercera opción económica desde la teoría de recursos de uso común. Recuperado en: http://www.scielo.org.co/pdf/ecos/v17n37/ v17n37a6.pdf

Ojeda, P. "La articulación de los acueductos comunitarios rurales, una estrategia para su fortalecimiento. Estudio de caso del proceso de articulación de los acueductos comunitarios del Valle del Cauca. IMCA. Buga - Valle del Cauca." 2014, p. 15. Recuperado en: http://semillas.org.co/es/revista/acueductoscomunitarios-alternativos-para-el-manejo-sostenible-del-aguay-la-sequ

Ojeda P. (2015). Alianza por el fortalecimiento de la gestión comunitaria del agua. Pontificia Universidad Javeriana. Recuperado en: https://www.javerianacali.edu.co/alianza-por-el-fortalecimiento-de-la-gestion-comunitaria-del-agua

Presidencia de la República. (2016). Decreto 1898 de noviembre de 2016. Por el cual se adiciona el Título 7, Capítulo 1, a la Parte 3, del Libro 2 del Decreto 1077 de 2015, que reglamenta parcialmente el artículo 18 de la Ley 1753 de 2015, en lo refe- rente a esquemas diferenciales para la prestación de los servicios de acueducto, alcantarillado y aseo en zonas rurales. Recuperado en: http://es.presidencia.gov.co/normativa/normativa/DECRETO\%201898\%20DEL\%2023\%20DE\%20NOVIEMBRE\%20DE\%202016.pdf

Presidencia de la República de Colombia. (2000). Decreto 421 de 2000, Numeral 4 del artículo 15. Por el cual se reglamenta el numeral 4 del artículo 15 de la Ley 142 de 1994, en relación con las organizaciones autorizadas para prestar los servicios públicos de agua potable y saneamiento básico en municipios menores, zonas rurales y áreas urbanas específicas. Recuperado en: http://www.alcaldiabogota.gov.co/sisjur/normas/Norma1. jsp?i=4639

Smits, S., Tamayo, S. P., Ibarra, V., Rojas, J., Benavidez, A. \& Bey, V. (2012). Gobernanza y sostenibilidad de los sistemas de agua potable y saneamiento rurales en Colombia. Banco Interamericano de Desarrollo. Jul. 15, 2012, pp.24-25. Recuperado en:

https://publications.iadb.org/handle/11319/3137\#sthash.vIrsBLEe.dpuf

Superintendencia de Servicios Públicos Domiciliarios. Acueducto y Alcantarillado. (2014). Acueducto y alcantarillado. Recuperado en: http://www.superservicios.gov.co/Acueducto-Alcantarillado-Aseo/Acueducto-y-alcantarillado

Unión Internacional para la Conservación de la Naturaleza, UICN. (2015). Acueductos comunitarios: una mirada a los administradores locales del agua. Recuperado en: https://www.iucn.org/ es/content/acueductos-comunitarios-una-mirada-los-administradores-locales-del-agua

Vanegas, Y., Vera L. \& Torres Ortega, J. (2014). Evaluación de la huella hídrica del lirio japonés. Recuperado en: http://hemeroteca.unad.edu.co/index.php/publicaciones-e-investigacion/ article/view/1285/1621 


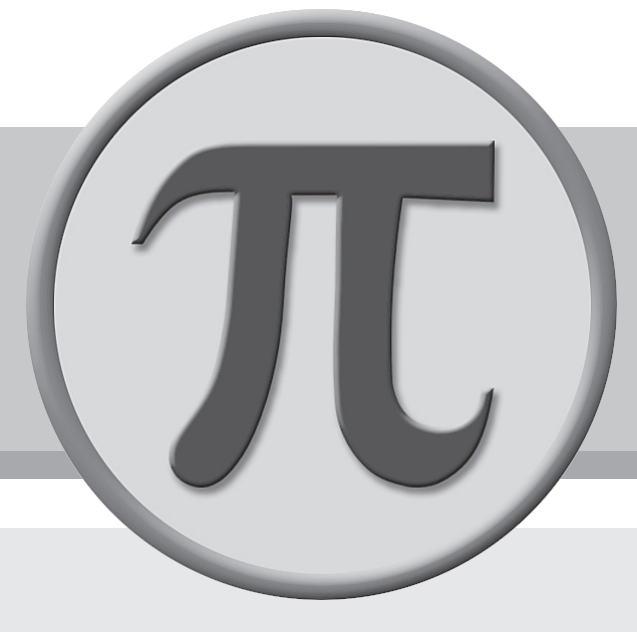


